\title{
Novel insertion mutation (R1822_E1823dup) in MYH6 coiled-coil domain causing familial atrial septal defect
}

\author{
Shufang Huang ${ }^{1}$, Yueheng $\mathrm{Wu}^{2}$, Shaoxian Chen ${ }^{1}$, Yongcha Yang ${ }^{3}$, Yonghua Wang ${ }^{4}$, \\ Haiping Wang ${ }^{1}$, Ping $\mathrm{Li}^{4}$, Jian Zhuang $^{2}$, and Yu Xia ${ }^{5}$ \\ ${ }^{1}$ Guangdong Provincial People's Hospital, Guangdong Academy of Medical Sciences \\ ${ }^{2}$ Guangdong Cardiovascular Institute \\ ${ }^{3}$ Guangdong Cardiovascular Institute, Guangdong General Hospital, Guangdong Academic \\ of Medical Science \\ ${ }^{4}$ Guangdong Provincial People's Hospital \\ ${ }^{5}$ The First Affiliated Hospital of Jinan University
}

November 6, 2020

\begin{abstract}
Atrial septal defect, secundum (ASD II, OMIM: 603642) is the second common congenital heart defect (CHD) in China. However, the genetic etiology of familial ASD II remains elusive. Using whole-exome sequencing (WES) and Sanger sequencing, we identified a novel myosin heavy chain 6 (MYH6) gene insertion variation, NM_002471.3:c.5465_5470dup (R1822_E1823dup), in a large Chinese Han family with ASD II. The variant R1822_E1823dup co-segregated with the disease in this family with autosomal dominant inheritance. The insertion variant located in the coiled-coil domain of the MYH6 protein, which is highly conserved across homologous myosin proteins and species. In transfected myoblast C2C12 cell lines, the MYH6 R1822_E1823dup variant significantly impaired myofibril formation and increased apoptosis but did not significantly reduce cell viability. Furthermore, molecular simulations revealed that the R1822_E1823dup variant impaired the myosin $\alpha$-helix, increasing the stability of the coiled-coil myosin dimer, suggesting that this variant has an effect on the coiled-coil domain self-aggregation. These findings indicate that R1822_E1823dup variant plays a crucial role in the pathogenesis of ASD II. Collectively, our findings expand the spectrum of MYH6 variations associated with familial ASD II and may provide a molecular basis in genetic counseling and prenatal diagnosis for this Chinses family.
\end{abstract}

Novel insertion mutation (R1822_E1823dup) in MYH6 coiled-coil domain causing familial atrial septal defect Shufang Huang2,3+, Yueheng Wu4,5,6+, Shaoxian Chen5,6, Yongchao Yang4,5, Yonghua Wang2,3, Hai Ping Wang2,3, Ping Li2,3, Jian Zhuang4,5, Yu Xia1,2,4

${ }^{1}$ Department of Cardiovascular Surgery, The First Affiliated Hospital, Jinan University, Guangzhou, China ${ }^{2}$ Prenatal Diagnosis Center, Guangdong Provincial People's Hospital, Guangdong Academy of Medical Sciences, Guangzhou, China

${ }^{3}$ Department of Obstetrics and Gynecology, Guangdong Provincial People's Hospital, Guangdong Academy of Medical Sciences, Guangzhou, China

${ }^{4}$ Department of Cardiovascular Surgery of Guangdong Provincial Cardiovascular Disease Institute, Guangdong Provincial People's Hospital, Guangdong Academy of Medical Sciences, Guangzhou, China ${ }^{5}$ Guangdong Provincial Key Laboratory of South China Structural Heart Disease, Guangdong Provincial People's Hospital, Guangdong Academy of Medical Sciences, Guangzhou, China 
${ }^{6}$ Research Department of Medical Sciences, Guangdong Provincial People's Hospital, Guangdong Academy of Medical Sciences, Guangzhou, China

\title{
Correspondence
}

Yu Xia

Department of Cardiovascular Surgery,

The First Affiliated Hospital, Jinan University, Guangzhou,

613\# West Huangpu Avenue,

Tianhe District, Guangzhou, China, 510630.

E-mail: line_user@163.com

+Shufang Huang and Yueheng Wu contributed equally to this work.

\section{Funding information}

This project was supported by the following funders: National Natural Science Foundation for Young Scientists of China, Grant Numbers: 81700223; Project founded by China Postdoctoral Science Foundation, Grant Numbers: 2018M633020, (P.I.: Yu. Xia).

\begin{abstract}
Atrial septal defect, secundum (ASD II, OMIM: 603642) is the second common congenital heart defect (CHD) in China. However, the genetic etiology of familial ASD II remains elusive. Using whole-exome sequencing (WES) and Sanger sequencing, we identified a novel myosin heavy chain 6 ( $M Y H 6$ ) gene insertion variation, NM_002471.3:c.5465_5470dup (R1822_E1823dup), in a large Chinese Han family with ASD II. The variant R1822_E1823dup co-segregated with the disease in this family with autosomal dominant inheritance. The insertion variant located in the coiled-coil domain of the MYH6 protein, which is highly conserved across homologous myosin proteins and species. In transfected myoblast $\mathrm{C} 2 \mathrm{C} 12$ cell lines, the MYH6 R1822_E1823dup variant significantly impaired myofibril formation and increased apoptosis but did not significantly reduce cell viability. Furthermore, molecular simulations revealed that the R1822_E1823dup variant impaired the myosin $\alpha$-helix, increasing the stability of the coiled-coil myosin dimer, suggesting that this variant has an effect on the coiled-coil domain self-aggregation. These findings indicate that R1822_E1823dup variant plays a crucial role in the pathogenesis of ASD II. Collectively, our findings expand the spectrum of $M Y H 6$ variations associated with familial ASD II and may provide a molecular basis in genetic counseling and prenatal diagnosis for this Chinses family.
\end{abstract}

\section{KEYWORDS}

atrial septal defect, $M Y H 6$, myofibril formation, apoptosis, whole exome sequencing

\section{INTRODUCTION}

Congenital heart defects (CHDs) are the most common life-threatening birth defect in China (Zhao et al., 2019; Sun et al., 2017). Atrial septal defect, secundum (ASD II, OMIM: 603642), which accounts for $20 \%$ to $25 \%$ of all CHD patients, is the second subtype of CHDs anatomically characterized by an interatrial communication within the region of the true septum, due to the absence of the septum primum and the superior interatrial fold (Dong et al., 2019; Naqvi et al., 2018). ASD II is often related with other intraand/or extra-cardiac anomalies. The pathophysiology of ASD II involves a right-to-left shunt at the atrial level resulting in enlargement of the right side of the heart and pulmonary hypertension. Patients with ASD often require transcatheter or surgical closure and have an overall excellent prognosis (Houeijeh et al., 2018).

The phenotype of ASD is associated with various cardiac and noncardiac defects, and genetic syndromes (Gilboa et al., 2016). In recent years, whole-exome sequencing (WES) technology has been utilized to explore CHD-causing variations (Jin et al., 2017). Emerging evidence has suggested that pathogenic copy number 
variants (CNVs) and gene mutations are responsible for the genetic etiology of familial ASD II (Hussein et al., 2018; Hu et al., 2018). Rare pathogenic CNVs at recurrent loci, such as 5q35.1 (Baekvad-Hansen et al., 2006), 8p23.1 (Pope et al., 2012) and 12q24 (Kimura et al., 2015), as well as numerous pathogenic gene variations in transcription factors, such as NK2 homeobox 5 (NKX2-5, OMIM: 600584) (Zakariyah et al., 2015), GATA binding protein 4 (GATA4, OMIM: 600576) (Liu et al., 2018) and T-box transcription factor 5 (TBX5, OMIM: 142900) (Behiry et al., 2019), have been associated with familial ASD II. Nevertheless, congenital ASD II is a genetically heterogeneous disease and the genetic basis for ASD II remains elusive.

The myosin heavy chain 6 ( YYH6 ) gene (14q11.2; OMIM: 160710) encodes the alpha heavy chain subunit of cardiac myosin-6 (MYH6), which belongs to the family of myocardial sarcomeric myosin proteins (Taylor et al., 2015). Two MYH6 monomers undergo self-assembly of the cardiac myosin dimer to form thick filaments. MYH6 contains a conserved head motor domain at the N-terminus, a neck region, and a coiled-coil domain at the C-terminus. The head motor domain has actin-activated $\mathrm{Mg}^{2+}$ - ATPase activity. The coiled-coil domain contains distinctive highly heptad repeat sequences in the form of $(\operatorname{abcdefg})_{\mathrm{n}}$, characteristic for $\alpha$-helical coiled coils, and four skip residues (Taylor et al., 2015). Residues at the "a" and "d" positions are invariably hydrophobic, and their interactions are critical for $\alpha$-helical coiled-coil stability ${ }^{17}$. Residues at the "e" and "g" positions involve electrostatic interactions, which further allow for structure stability. The remaining surface residues are responsible for forming thick filaments (Viswanathan et al., 2017).

Small changes in the level of MYH6 protein expression can dramatically influence cardiac development, embryonic survival, and heart function. MYH6 mutations display a wide spectrum of disorders, including ASD (Ching et al., 2005; Granados-Riveron; Posch et al., 2010; Arrington et al., 2012; Liu et al., 2018; Hu et al., 2018; Razmara et al., 2018; Xia et al., 2019), dilated cardiomyopathy (DCM) (Merlo et al., 2013), hypertrophic cardiomyopathy (HCM) (Carniel et al., 2005), hypoplastic left heart syndrome (HLHS) (Theis et al., 2015; Tomita-Mitchell et al., 2016) and sick sinus syndrome (SSS) (Ishikawa et al., 2015; Lam et al., 2015), suggesting that cardiac sarcomeric proteins have a profound impact on the cardiac septal structure and myocardial and electrophysiological disorders.

In this study, we report on a novel pathogenic insertion variant in $M Y H 6$ in a four generation Chinese Han family with ASD II by WES. We also discuss plausible mechanisms for the cause of ASD II through molecular and functional analyses.

\section{MATERIALS AND METHODS}

\subsection{Study subjects}

This study protocol was approved by Research Ethics Committee of the Guangdong Provincial People's Hospital (No. 2017073). Proband III-3 sought medical advice in the Department of Congenital Heart Disease Surgery and Prenatal Diagnosis Center of the Guangdong Provincial People's Hospital in July 2017. Family histories were obtained by interviewing family members. Written Informed consents for medical examination and genetics research were obtained from all family members. All family members received a complete medical examination. Available clinical data, including medical records, family history, echocardiograms, electrocardiograms, cardiac catheterization reports, and surgical records, were reviewed. Each of the cardiac anomalies of the patients were confirmed during intervention or operation.

Approximately $4.0 \mathrm{~mL}$ of peripheral venous blood specimen was collected from the available family members and. Genomic DNA was isolated from peripheral blood using Gentra Puregene blood kit (QIAGEN, Santa Clara, CA, USA). A cohort of 176 unrelated healthy controls were selected from our local database.

\subsection{Conventional cytogenetic analysis}

Peripheral blood $(2.0 \mathrm{ml}$ ) from patient III-3 and his son IV-2 was collected and subjected to lymphocyte culture. Cytogenetic analysis was performed by conventional G-Banded techniques at the level of 550 bands according to standard cytogenetic protocols.

\subsection{Chromosomal microarray analysis (CMA), pathogenic CNV evaluation and validation}


DNA copy number was assessed by CytoScan HD array platform (Affymetrix, Santa Clara, CA, USA) according to the manufacturer's protocol. CNV gains or losses were filtered by Chromosome Analysis Suite (ChAS 2.0). The CNV were annotated by the Genome Reference Consortium (GRC) human reference genome version GRCh37/hg19. The pathogenicity of the CNVs was assessed by the American College of Medical Genetics and Genomics (ACMG) standards and guidelines ${ }^{32}$.

\subsection{Whole exome sequencing and variant analysis}

To systematically search for disease-causing gene mutations, exome sequencing in three affected individuals (III-2, III-3, and III-13) and one unaffected individual (IV-1) was performed. Exome capture was performed by SureSelect Human All Exon V6 (Agilent Technologies, Santa Clara, CA, USA) according to the manufacturer's protocol. The qualified libraries were applied to $2 \times 150$ bp paired-end sequencing on the Illumina HiSeq X-ten platform (Illumina, San Diego, CA, USA). FASTQ files were aligned to the GRCh37/hg19 by BWA v0.7.13 (Li et al., 2009; Kent et al., 2002). The aligned bam format files were first sorted by samtools (1.3) and then duplicates were flagged using Picard (2.2.4) (Li et al., 2009). Reads were locally realigned and base qualities were recalibrated, by GATK v3.5. Finally, mapping statistics that included depth and coverage were generated from recalibrated files by in-house perl/python scripts and BEDTools (2.16.1).

Variants, including single nucleotide variants (SNVs) and indels, were genotyped from recalibrated BAM files using the multi-sample processing mode of the HaplotypeCaller tool from GATK. Variant quality score recalibration (VQSR) was used to reduce false positives of variant calling. SNVs and indels were annotated using ANNOVAR software (Wang et al., 2010) against multiple databases including the gnomAD (https://gnomad.broadinstitute.org/), 1000G (http://www.1000genomes.org) and Exome Sequencing Project (ESP) (https://esp.gs.washington.edu). The pathogenicity of the variants was assessed according to the ACMG standards and guidelines (Richards et al., 2015).

\subsection{Sanger sequencing}

To confirm potential causative variants in the family, Sanger sequencing was performed, using an ABI 3500 sequencer (Applied Biosystems, Foster City, CA, USA). Primer sequences for the variant in the MYH6 gene (NM_002471) were: F 5'- ACCATTAAGGACCTGCAGCAC -3' and R 5'- ATTTGCTGGAAAAACTCATAGACTC -3'. Once a variant was confirmed, all available family members were screened to determine variant segregation within the family.

\subsection{Plasmids construction and site-directed mutagenesis}

Full-length human $M Y H 6$ cDNA and its mutant were PCR-amplified from IMAGE clone 14406689 (GenBank Accession number BC132667.1) and cloned into the adenoviral vector GV138 (Genechem) with a 3FLAG-tag at an AgeI/NheI site. Adenoviruses were generated by cotransfecting HEK293 cells with Ad-MHY6 WT or Ad-MHY6 R1822_E1823dup constructs and packaged constructs.

\subsection{Cell infection and imaging}

To investigated the mutations of $M Y H 6$ affect formation of myofibrils, we use mouse C2C12 model cell line. Owing to the $\mathrm{C} 2 \mathrm{C} 12$ cells can differentiate into muscle fibre-like cells and present sarcomeric structure, this myoblast has been used to study human cardiac myosin function and associated disease (Wang et al., 2003; Granados-Riveron et al., 2010). Cell transfection and imaging were performed as previously described (Wang et al., 2003; Granados-Riveron et al., 2010). Briefly, mouse C2C12 myoblasts were cultured in Dulbecco's modified Eagle's medium (DMEM) supplemented with 10\% fetal bovine serum (FBS; Invitrogen, Karlsruhe, Germany) (Wang et al., 2003; Granados-Riveron et al., 2010). For cell infection, cells were seeded on precoated glass coverslips in 12-well plates. Cells were infected with Ad-MHY6 WT or Ad-MHY6 R1822_E1823dup. To induce differentiation, the infected cells were transferred to differentiating medium and further cultured for 7 days in differentiation medium (media is made up of DMEM $+1 \%$ horse serum), which was changed daily after infection. On day 8, cells on coverslips were fixed in $4 \%$ paraformaldehyde for 20 min. Laser scanning confocal microscopy was used by Leica TCS SP5 (Leica Microsystems, Heidelberg, Germany) using a $400 \times 1.4$ oil objective. 


\subsection{Structural analysis of MYH6 mutations}

The three-dimensional structure of wild type MYH6 (1779-1857) was directly cut out of the corresponding domain (1777-1855) in Chains A and B of MYH7 (4XA6, resolution: $3.42 \AA$ ) with homological identity of $100 \%$. The mutant MYH6 was derived from homology modeling performed by SWISS-MODEL (https://swissmodel.expasy.org) through insertion of two amino acids, arginine and glutamic acid, into tween Glu1823 and Leu1824. The model structure was then optimized in a 100 ps molecular dynamics (MD) simulation. For each structure, 10 ns MD simulation was performed using GROMACS version 2016.4 (Abraham et al., 2015) with AMBER ff99SB-ILDN force field and TIP3P ${ }^{41}$ water model with SPC216 ${ }^{42}$ solvent configuration.

\subsection{Cell viability assay and apoptosis analysis}

Cell viability was analyzed using 3-(4,5-dimethylthiazol-2-yl)-2,5-diphenyl-tetrazolium bromide (MTT) assay after transfection 2 days. PE Annexin V Apoptosis Detection Kit I (BD Pharmingen, USA) was used to detect apoptotic cells. Briefly, cells were resuspended in binding buffer at a concentration of $1 \times 10^{6}$ cells $/ \mathrm{mL}$. One hundred microliters of the solution $\left(1 \times 10^{5}\right.$ cells $)$ was transferred to a $5 \mathrm{~mL}$ culture tube. Five microliters of 7 -AAD and $5 \mu \mathrm{PE}$ Annexin $\mathrm{V}$ were added and incubated at $25^{\circ} \mathrm{C}$ for $20 \mathrm{~min}$ in the dark. Samples were analyzed by flow cytometry (BD, FACSverse, USA) within 1 hour.

\subsection{Statistical analysis}

Statistical analysis was performed with SPSS 23.0 (IBM, USA). Results are presented as mean \pm standard deviation. ANOVA, $\chi^{2}$ test was employed for comparisons between groups. A $p$ value of $<0.05$ was considered statistically significant.

\section{RESULTS}

\subsection{Clinical characteristics of families}

The four generation family pedigree exhibiting ASD II (Figure 1 A) was obtained. All the enrolled affected family members were previously diagnosed with ASD II at the time of enrollment.

In the family (Table 1), the proband was patient III-3, who was diagnosed with ASD II at 9 years old and underwent surgical closure of the defect at 10 years old. The proband's sister, patient III-2, had minor ASD II that did not require closure. Notably, the proband's son, patient IV-2, was diagnosed with ASD II, ostium primum ASD, mitral valve cleft, and mitral valve regurgitation. Ostium primum ASD that was characterized by within the spectrum of the atrioventricular septal defects (AVSD) with communication at the atrial level only. At the age of 2 years, patient IV-2 underwent surgical correction for ostium primum ASD and ASD II.

Patient I-2 presented with a heart murmur and her medical history was unavailable. Patient II-2 and patient II-7 died due to cardiac accidents at 70 and 58 years old, respectively. Patient II-3 had ASD II and underwent surgery to repair the defect at 55 years old. His daughter, patient III-6, was diagnosed with ASD II when she was eight. At the age of 9 years, she underwent interventional closure of the defect. Patient II-9 and patient III-13 were diagnosed with ASD II and underwent surgical and interventional closure of the defect at 48 and 7 years old, respectively.

\subsection{Conventional G-banded cytogenetic analysis and CMA findings in the proband and his son}

Due to the obvious family history of the proband, we initially hypothesized that chromosome abnormality or CNV was likely responsible for this condition. Conventional G-banded cytogenetic analysis and CMA were performed for patient III-3 and his son, patient IV-2. However, no clinically significant differences were found in these two patients. Based on these results, we excluded the possibility of chromosome abnormalities or pathogenic CNVs and focused on gene mutations instead.

\subsection{Exome sequence analysis and segregation of variants}


Exome sequencing of four individuals (III-2, III-3, III-13 and IV-1) generated a mean of 73,230,394 pairs of sequenced reads totally, and had an average depth of $129.89 \times$. In total, $93.50 \%$ of sequenced reads passed the quality assessment. Qualified reads were mapped to $99.40 \%$ of the human reference genome. After filtering, 799 single nucleotide substitutions and 121 indel variants were shared by three patients (III-2, III-3 and III13). Preliminarily, by our screening strategy, 66 candidate genes were screened out, in which a heterozygous NM_002471.3:c.5465_5470dup, R1822_E1823dup variant of MYH6(NM_002471.3) was observed in proband III-3 but not in IV-1.

\subsection{Identification of a pathogenic mutation and cosegregation analysis}

The R1822_E1823dup variant has not been reported in the 1000G, ESP6500, gnomAD, HGMD or ClinVar databases, and has not been previously described in CHD patients or control subjects. The residue at codon 1822-1826 in the coiled-coil domain of MYH6 is highly conserved in all available vertebrate myosin sequences (Figure 1 C). Bioinformatics programs predicted R1822_E1823dup to be pathogenic across all prediction algorithms. $M Y H 6$ is a known ASD-causative gene and plays an important role in cardiac development or structure/function.

Sanger sequencing confirmed the same MYH6 R1822_E1823dup variant in all patients (II-3, II-9, III-2, III-3, III-6, III-13 and IV-2), but not in the healthy individuals (II-4, II-5, II-8, II-10, II-11, II-12, II-13, II-14, III-8, III-9, III-12, III-14, III-15, IV-1, IV-3, IV-4, IV-7 and IV-8) and 176 controls (Figure 1 B). The heterozygous MYH6 R1822_E1823dup completely cosegregated with ASD in the family. Patient IV-2 who harbored this variant inherited it from patient III-3. Patients III-2 and III-3 carried the variant, which might be inherited from their mother (II-2). The genetic information for patients II-2 and II-7 was unavailable.

Taken together, the sequencing results indicate that the R1822_E1823dup variant may contribute to the pathogenesis of ASD in this family.

\subsection{Mutations of $M Y H 6$ affect formation of myofibrils}

To investigate whether the R1822_E1823dup change alters human MYH6 -mediated formation of myofibrils, we examined the structural changes of the $M Y H 6$ variant on myofibrillar organization in transient transfected $\mathrm{C} 2 \mathrm{C} 12$ cells. Following the methods of Javier et al. and Wang et al. ${ }^{23,24}$, transfected cells were classified into three groups: Striated I, Striated II and non-striated, according to myofibrillar organization. Striated I class cells display definite myofibrils. In this group, cells that paralleling to each other along the their long axis (Figure 2 A). Striated II class cells contain a mixture of non-striated myofilaments interspaced with misaligned and thin striated myofibrils (Figure $2 \mathrm{~B}$ ). Non-striated cells contain brightly fluorescent speckles and lack organized repeating sarcomeres, that is characteristic of myofibrils (Figure $2 \mathrm{C}$ ). We imaged more than 60 cells from each group in three independent transfection experiments, which are summarized in Figure 2 D. The R1822_E1823dup variant significantly reduced the striated patterns (I and II), with an increase of a non-striated pattern simultaneously $(p<0.05)$, suggesting that this mutation dramatically decreased myofibril arrays.

\subsection{In silico protein modeling of MYH6 protein}

The molecular architecture of MYH6 protein is well-established, enabling protein modeling to predict the effects of mutation on structure and function. The R1822_E1823dup variant is located in the 'd' and 'e' position in the heptad repeat, respectively, and promotes the exposure of the side groups of Glu1823 and $\operatorname{Arg} 1823^{\Delta}$ in the 'c' and 'd' position. This insertion of two amino acids is connected to two flanks in the form of a random-coil, instead of $\alpha$-helix. The long helix peptide chain of mutant MYH6 is divided into three short helix regions between the 1812 and 1857 amino acids. The volume of the dimer is clearly expanded.

An insertion in the 1823-1824 site results in the disruption of hydrogen bonds between peptide chains, such as $\operatorname{Arg} 1834^{\mathrm{A}}-\mathrm{Asn} 1835^{\mathrm{B}}$ and Gln1813 ${ }^{\mathrm{A}}-\mathrm{Glu} 1818^{\mathrm{B}}$. However, some new hydrogen bonds (Arg1820A-Gln1825B, Ser1838A-Asn1835B and Met1842A-Ser1845B) are created, which further strengthens the integration between chains, enhancing the stability of the dimer and decreasing its motility (Figure $3 \mathrm{~A}, \mathrm{~B}$ ). 


\subsection{Cell viabilityu}

Since the R1822_E1823dup variant disrupted the coiled-coil domain, to determine whether cell viability was affected, an MTT assay was used. There was no significant difference in cell viability between the MYH6-R1822_E1823dup group and the MYH6-WT group ( $p>0.05)$ (Figure 4 D).

\subsection{Apoptosis analysis}

C2C12 cells undergoing apoptosis after transfection for 48 and $72 \mathrm{~h}$ were detected using the Annexin V flow cytometry assay (Figure $4 \mathrm{~A}-\mathrm{C}$ ). Annexin-V positive cells showed that the number of apoptosis cells in the MYH6-R1822_E1823dup group was significant higher than that of control group $(p<0.05)$. These results suggest that the R1822_E1823dup variant can increase apoptosis of C2C12 muscle cells.

\section{DISCUSSION}

In this study, we describe a novel pathogenic variant of MYH6, R1822_E1823dup, which cosegregates with ASD II in a large Chinese Han family in an autosomal dominant manner. Notably, although the predominant defect in this family is ASD II, we observed PAVSD in patient IV-2, which is a significant intrafamilial phenotypic variation that has commonly been described in previous studies (Posch et al., 2011; GranadosRiveron et al., 2010). To study this pleiotropism, we performed the whole genome sequence (WGS) assembled by PacBio sequencing. However, WGS combined with the WES, we not find the genetic cause of ostium primum ASD, suggesting that a novel gene, epigenetic modifications or environmental factors may be involved in the pathogenesis of ostium primum ASD. These phenomena highlight the genetic heterogeneity of CHD and complexity of identifying CHD-causing gene variants.

MYH6 is predominantly expressed in the fetal atrium and is closely related to atrial septum development. Rutland et al. ${ }^{43}$ showed that MYH6 knockdown lead to developmental failure of atrial septum during gallus embryo development. Several studies have shown some variants in the head motor domain of MYH6 protein may impact its myofibril formation, which may be associated with ASD. Previous studies have found that both MYH6 C539R and K543R variants are situated in the highly conserved actin-myosin interface and are predicted to impair the interaction of $\alpha$-myosin with actin (Posch et al., 2011). Granados-Riveron et al. (Granados-Riveron et al., 2010) reported that MYH6 p.A230P and p.H252Q mutations, which are associated with ASD, significantly disrupt myofibril formation and enhance myofibril assembly, respectively. These studies proposed that disturbed myofibril formation might result in an inhibition of atrial septum genesis and provide a novel potential mechanism between ASD and myofibril formation associated MYH6 mutations. Based on the studies above, we hypothesized that MYH6 mutations coiled-coil domain that influence myofibril formation may be related to ASD. To assess this possibility, we examined myofibril formation similar to previous studies. As expected, our results revealed that the R1822_E1823dup variant myosin-6 has lost its ability to form myofibrils in differentiated $\mathrm{C} 2 \mathrm{C} 12$ cells significantly, suggesting pathogenesis of the R1822_E1823dup variant occurs by affecting myofibril formation.

Currently, a number of pathogenic variants located in the MYH6 gene have been associated with ASD, most of which are located in the coiled-coil domain (Taylor et al., 2015; Blue et al., 2014). The L1824delR1822_E1823dupL insertion occurs in the residues at the ' $d$ ' and ' $e$ ' positions of the MYH6 coiled-coil domain, which is highly conserved across sarcomeric myosin families. Our molecular simulations also revealed that the structure of the insertion of the amino acids Glu1823 $\Delta$ and $\operatorname{Arg} 1824 \Delta$ was connected to two flanks in the form of a random-coil, rather than an $\alpha$-helix. Furthermore, the occupied insertion further pushed against the $\alpha$-helix backward, causing the connection between 1842 and 1840 to transform into a randomcoil. The R1822_E1823dup clearly increased volume expansion of the dimer and impaired the $\alpha$-helix, and divided the long helix peptide chain into three short helix regions between 1812 and 1857. In addition, the R1822_E1823dup variant can increase the stability of the coiled-coil mediated dimerization of myosin 6 , due to creation of new hydrogen bonds between the two chains. Taken together, these results further support the idea that the R1822_E1823dup variant affects myofibril formation.

Several studies have shown that imbalance of apoptosis and cell proliferation contributes to the pathogenesis 
of ventricular septal defect (Wu et al., 2016; Wang et al., 2015). In our study, we observed that the R1822_E1823dup variant promoted C2C12 apoptosis without affecting the cell viability, suggesting MYH6 may be involved in atrial septal development by affecting the balance between apoptosis and proliferation. However, the relationship between myofibril formation and apoptosis is still unclear, and further studies are needed to uncover precise mechanism of apoptosis.

Our study also illustrates that WES is an accurate and reliable technique for identifying pathogenic variants related to cardiac defects in pedigree. Additionally, we did find no positive clinical results in this large ASD II pedigree by conventional G-banded cytogenetic analysis and CMA, however, these negative results helped us exclude chromosome abnormalities or pathogenic CNV and allowed us to focus on gene mutations. These combined clinical genetic data will facilitate molecular diagnosis and genetic counseling to assess the recurrence risk and prognosis of newborns.

This study has a few limitations. First, the effects of the R1822_E1823dup variant on sarcomere kinesiology function were not further analysed. Second, we did not obtain phenotypic data in other species such as the mouse, since Myh6 is not expressed in the mouse atrium. The functional link between sarcomere formation and atrial septum development requires further research.

In summary, we identified a novel MYH6 heterozygous insertion variant, R1822_E1823dup, in a large Chinese Han family with ASD II by WES. The R1822_E1823dup variant significantly impaired myofibrillar organization and increased apoptosis in $\mathrm{C} 2 \mathrm{C} 12$ cells, suggesting that this mutation plays an important role in familial ASD II. These findings expand our current knowledge of the mutation spectrum of the MYH6 gene associated with ASD and offer further insight into providing appropriate diagnosis and genetic counseling of affected families.

\section{ACKNOWLEDGMENTS}

We sincerely thank all of the patients and their family members for their enthusiasm and continued participation in this study. We would also like to thank the clinicians and physicians for sending the blood samples. We thank Dr. Shougang Song, Dr. Shan Wang and Dr. Lili Zang (Beijing Novogene, Inc) for their technical assistance in WES. We thank Dr. Ziying Zhang (Guangzhou Yinfo Information Technology Co, Ltd) for his advice in molecular dynamics simulations.

\section{CONFLICT OF INTERESTS}

All authors declare no conflict of interest.

\section{DATA AVAILABILITY STATEMENT}

The data that support the findings of this study are available from the corresponding author upon reasonable request.

\section{References}

Abraham M. J., Murtola T., Schulz R., Páll S., Smith J. C., Hess B., Lindahl E. GROMACS: High performance molecular simulations through multi-level parallelism from laptops to supercomputers. SoftwareX. 2015;1-2:19-25.

Arrington C. B., Bleyl S. B., Matsunami N., Bonnell G. D., M Otterud B. E., Nielsen D. C., .. Bowles N. E. (2012). Exome Analysis of a Family With Pleiotropic Congenital Heart Disease. Circulation. Cardiovascular Genetics, 5(2), 175-182. DOI: 10.1161/CIRCGENETICS.111.961797

Baekvad-Hansen M., Tümer Z., Delicado A., Erdogan F., Tommerup N., Larsen L. A. (2006). Delineation of a 2.2 Mb Microdeletion at 5q35 Associated With Microcephaly and Congenital Heart Disease.American Journal of Medical Genetics Part A , 1, 140(5), 427-33. DOI: 10.1002/ajmg.a.31087

Behiry E. G., Al-Azzouny M. A., Sabry D., Behairy O. G., Salem N. E. (2019). Association of NKX25, GATA4, and TBX5 Polymorphisms With Congenital Heart Disease in Egyptian Children. Molecular 
Genetics and Genomic Medicine, 7(5), e612.

Blue G. M., Kirk E. P., Giannoulatou E., Dunwoodie S. L., Ho J. W. K., Hilton D. C. K., .. Winlaw D. S. (2014). Targeted next-generation sequencing identifies pathogenic variants in familial congenital heart disease. Journal of the American College of Cardiology, 14, 64, 2498-2506. DOI: 10.1016/j.jacc.2014.09.048

Berendsen H. J., Postma P. M., Gunsteren W.F. (1981). Interaction models for water in relation to protein hydration. Intermolecular Forces, 331-342.

Carniel E., Taylor M. R. G., Sinagra G., Lenarda A. D., Ku L., Fain P. R., .. Mestroni L. (2005). Alphamyosin Heavy Chain: A Sarcomeric Gene Associated With Dilated and Hypertrophic Phenotypes of Cardiomyopathy. Circulation , 112(1), 54-59. DOI: 10.1161/CIRCULATIONAHA.104.507699

Ching Y. H., Ghosh T. K., Cross S. J., Packham E. A., Honeyman L., Loughna S., .. Brook J. D. (2005). Mutation in Myosin Heavy Chain 6 Causes Atrial Septal Defect. Nature Genetics , 37(4), 423-428. DOI: $10.1038 / \operatorname{ng} 1526$

Dong Y. Y., Zhang Y.T., Tong S. L., Jiang Z.Y., Xu Z. W., Li X. Y., Wang W. (2019). Analysis of the Seasonal Trend of Congenital Heart Defects. The Journal of Pediatrics , 207, 29-33.e1.

Gilboa S. M., Devine O. J., Kucik J. E., Oster M. E., Riehle-Colarusso T., Nembhard W. N., ... Marelli A. J. (2016), Congenital heart defects in the United States: estimating the magnitude of the affected population in 2010. Circulation , 134, 101-9. DOI: 10.1161/CIRCULATIONAHA.115.019307

Granados-Riveron J. T., Ghosh T. K., Pope M., Bu'Lock F., Thornborough C., Eason J., .. Brook J. D. (2010). Alpha-cardiac Myosin Heavy Chain (MYH6) Mutations Affecting Myofibril Formation Are Associated With Congenital Heart Defects. Human Molecular Genetics , 19(20):4007-16. DOI: 10.1093/hmg/ddq315

Houeijeh A, Hascoët S., Bouvaist H., Hadeed K, Petit J., Godart F., Fraisse A. (2018). Transcatheter Closure of Large Atrial Septal Defects (ASDs) in Symptomatic Children With Device/Weight Ratio [?]1.5.International Journal of Cardiology , 15, 267, 84-87. DOI: 10.1016/j.ijcard.2018.05.069

Hussein I. R., Bader R.S., Chaudhary A. G., Bassiouni R., Alquaiti M., Ashgan F., .. Al Qahtani M. H. (2018). Identification of De Novo and Rare Inherited Copy Number Variants in Children With Syndromic Congenital Heart Defects. Pediatric Cardiology , 39(5), 924-940. DOI: 10.1007/s00246-018-1842-7

Hu P., Qiao F., Wang Y., Meng L., Ji X., Luo C., .. Xu Z. (2018). Clinical Application of Targeted NextGeneration Sequencing in Fetuses With Congenital Heart Defect. Ultrasound in obstetrics 83 gynecology, 52(2), 205-211. DOI: 10.1002/uog.19042

Ishikawa T., Jou C. J., Nogami A., Kowase S., Arrington C. B., Barnett S. M., .. Makita N. (2015). Novel Mutation in the $\alpha$-Myosin Heavy Chain Gene Is Associated with Sick Sinus Syndrome. Circulation. Arrhythmia and Electrophysiology, 8(2), 400-408. DOI: 10.1161/CIRCEP.114.002534

Jin S. C., Homsy J., Zaidi S., Lu Q. S., Morton S., DePalma S. R., .. Brueckner M. (2017). Contribution of rare inherited and de novo variants in 2,871 congenital heart disease probands. Nature Genetics , 49, 1593-1601. DOI: 10.1038/ng.3970

Jorgensen W. L., Chandrasekhar J., Madura J. D. (1983). Comparison of simple potential functions for simulating liquid water. The Journal of Chemical Physics, 79, 926.

Kent W. J., Sugnet C. W., Furey T. S., Roskin K. M., Pringle T. H., Zahler A. M., Haussler D. (2002). The human genome browser at UCSC.Genome Research , 12, 996-1006. DOI: 10.1101/gr.229102

Kimura M., Kikuchi A., Ichinoi N., Kure S. (2015). Novel TBX5 Duplication in a Japanese Family With Holt-Oram Syndrome.Pediatric Cardiololgy , 36(1), 244-247. DOI: 10.1007/s00246-014-1028-x

Lam L., Ingles J., Turner C., Kilborn M., Bagnall R. D., Semsarian C. (2015). Exome Sequencing Identifies a Novel Mutation in the MYH6 Gene in a Family With Early-Onset Sinus Node Dysfunction, 
Ventricular Arrhythmias, and Cardiac Arrest . HeartRhythm Case Reports , 30, 1(3), 141-145. DOI: 10.1016/j.hrcr.2015.01.022

Li H., Durbin R. (2009). Fast and accurate short read alignment with Burrows-Wheeler transform. Bioinformatics , 25, 1754-1760. DOI: 10.1093/bioinformatics/btp324

Li H., Handsaker B., Wysoker A., Fennell T., Ruan J., Homer N., .. 1000 Genome Project Data Processing Subgroup. (2009). The Sequence Alignment/Map format and SAMtools. Bioinformatics, 25, 2078-2079. DOI: $10.1093 /$ bioinformatics/btp352

Liu L., Yu R. (2018). A De Novo Novel Nonsense Mutation of GATA4 is Responsible for a Patient with Atrial Septal Defect. Pediatric Cardiology , 39(4), 852-853. DOI: 10.1007/s00246-018-1835-6

Liu Y., Cao Y., Li Y., Lei D., Li L., Hou Z. L., ... Jiang L. (2018). Novel Genetic Variants of Sporadic Atrial Septal Defect (ASD) in a Chinese Population Identified by Whole-Exome Sequencing (WES). Medical science monitor, 5, 24, 1340-1358. DOI: 10.12659/msm.908923

Manning M., Hudgins L. Professional Practice and Guidelines Committee. (2010). Array-based technology and recommendations for utilization in medical genetics practice for detection of chromosomal abnormalities.Genetics in Medicine , 12, 742-745. DOI: 10.1097/GIM.0b013e3181f8baad

Merlo M., Sinagra G., Carniel E., Slavov D., Zhu X., Barbati G., ... Taylor M. R. G. (2013). Familial Cardiomyopathy Registry. Poor Prognosis of Rare Sarcomeric Gene Variants in Patients With Dilated Cardiomyopathy. Clinical and translational science, 6(6), 424-8. DOI: 10.1111/cts.12116

Naqvi N., McCarthy K. P., Ho S. Y. (2018). Anatomy of the Atrial Septum and Interatrial Communications. Journal of Thoracic Disease, 10(Suppl 24), S2837-S2847. DOI: 10.21037/jtd.2018.02.18.

Pope K., Samanich J., Ramesh K. H., Cannizzaro L., Pan Q., Babcock M. (2012). Dextrocardia, Atrial Septal Defect, Severe Developmental Delay, Facial Anomalies, and Supernumerary Ribs in a Child With a Complex Unbalanced 8;22 Translocation Including Partial 8p Duplication.American Journal of Medical Genetics Part A , 158A(3), 641-647. DOI: 10.1002/ajmg.a.34431

Posch M. G., Waldmuller S., Müller M., Scheffold T., Fournier D., Andrade-Navarro M. A., ... Cemil Özcelik. (2011). Cardiac Alpha-Myosin (MYH6) Is the Predominant Sarcomeric Disease Gene for Familial Atrial Septal Defects. PLoS One, 6(12), e28872. DOI: 10.1371/journal.pone.0028872

Razmara E., Garshasbi M. (2018). Whole-exome Sequencing Identifies R1279X of MYH6 Gene to Be Associated With Congenital Heart Disease.BMC Cardiovascular Disorders, 18(1), 137. DOI: 10.1186/s12872018-0867-4

Richards S, Aziz N, Bale S., Bick D., Das S., Gastier-Foster J., ... ACMG Laboratory Quality Assurance Committee. (2015). Standards and guidelines for the interpretation of sequence variants: a joint consensus recommendation of the American College of Medical Genetics and Genomics and the Association for Molecular Pathology. Genetics in Medicine, 17, 405-424. DOI: 10.1038/gim.2015.30

Rutland C., Warner L., Thorpe A., Alibhai A., Robinson T., Shaw B., .. Loughna S. (2019). Knockdown of alpha myosin heavy chain disrupts the cytoskeleton and leads to multiple defects during chick cardiogenesis. Journal of anatomy, 214, 905-915. doi: 10.1111/j.1469-7580.2009.01079.x.

Sun P. F., Ding G. C., Zhang M. Y., He S. N., Gao Y., Wang J. H. (2017). Prevalence of Congenital Heart Disease Among Infants From 2012 to 2014 in Langfang, China. Chinese Medical Journal (English),130(9),10691073. DOI: $10.4103 / 0366-6999.204923$

Taylor K. C., Buvoli M., Korkmaz E. N., Buvoli A., Zheng Y.Q., Heinze N. T., ... Rayment I. (2015). Skip Residues Modulate the Structural Properties of the Myosin Rod and Guide Thick Filament Assembly.Proceedings of the National Academy of Sciences of the United States of America, 112(29), E3806-3815. DOI: $10.1073 /$ pnas. 1505813112 
Theis J. L., Zimmermann M. T., Evans J. M., Eckloff B. W., Wieben E. D., Qureshi M. Y., ... Olson T. M. (2015). Recessive MYH6 Mutations in Hypoplastic Left Heart With Reduced Ejection Fraction.Circulation: Cardiovascular Genetics , 8(4), 564-571. DOI: 10.1161/CIRCGENETICS.115.001070

Tomita-Mitchell A., Stamm K. D., Mahnke D. K., Kim M. S., Hidestrand P. M., Liang H. L., .. Mitchell M. E. (2016). Impact of MYH6 Variants in Hypoplastic Left Heart Syndrome. Physiological Genomics, 48(12), 912-921. DOI: 10.1152/physiolgenomics.00091.2016

Viswanathan M. C., Tham R. C., Kronert W. A., Sarsoza F., Trujillo A. S., Cammarato A., Bernstein S. I. (2017). Myosin Storage Myopathy Mutations Yield Defective Myosin Filament Assembly in Vitro and Disrupted Myofibrillar Structure and Function in Vivo. Human Molecular Genetics , 26(24), 4799-4813. DOI: $10.1093 / \mathrm{hmg} / \mathrm{ddx} 359$

Wang F., Wu Y. Q., Quon M. J., Li X. Z., Yang P. X. (2015). ASK1 mediates the teratogenicity of diabetes in the developing heart by inducing ER stress and inhibiting critical factors essential for cardiac development. American journal of physiology. Endocrinology and metabolism . 1, 309(5), E487-499. DOI: 10.1152/ajpendo.00121.2015

Wang K., Li M., Hakonarson H. (2010). ANNOVAR: functional annotation of genetic variants from highthroughput sequencing data. Nucleic Acids Research, 38, e164. DOI: 10.1093/nar/gkq603

Wang Q., Moncman C. L., Winkelmann D. A. (2003). Mutations in the motor domain modulate myosin activity and myofibril organization. Journal of Cell Science , 116, 4227-4238. DOI: 10.1242/jcs.00709

Wu Y., Zhou X., Huang X., Xia Q., Chen Z., Zhang X., .. Geng Y. J. (2016). Pax8 Plays a Pivotal Role in Regulation of Cardiomyocyte Growth and Senescence. Journal of cellular and molecular medicine, 20(4), 644-654. DOI: $10.1111 /$ jcmm.12779

Xia Y., Chen S., Yang Y. C., Wu Y. H., Huang S. F., Wang Y. H., .. Zhuang J. (2019). Novel Mutation in MYH6 in 2 Unrelated Chinese Han Families With Familial Atrial Septal Defect. Circulation: Genomic and precision medicine , 12(11), e002732. DOI: 10.1161/CIRCGEN.119.002732

Zakariyah A. F., Rajgara R. F., Veinot J. P., Skerjanc I. S., Burgon P. G. (2017). Congenital Heart Defect Causing Mutation in Nkx2.5 Displays in Vivo Functional Deficit. Journal of Molecular and Cellular, 105, 89-98. DOI: 10.1016/j.yjmcc.2017.03.003.

Zhao Q. M., Liu F., Wu L., Ma X. J., Niu C., Huang G. Y. (2019). Prevalence of Congenital Heart Disease at Live Birth in China. The Journal of Pediatrics , 204, 53-58. DOI: 10.1016/j.jpeds.2018.08.040

FIGURE 1. The large family carrying the heterozygous MYH6 R1822_E1823dup variant.

A : The proband is marked by an arrow. Full and open circles and squares indicate affected and normal individuals, respectively.B : The R1822_E1823dup variant in MYH6 confirmed by Sanger sequencing. The line segment represents the site of R1822_E1823dup.C : Conservation of the MYH6 R1822_E1823dup site in different species. The pink box represents the site of R1822_E1823dup in the amino acid sequence of different species, which is highly conserved. The inserted two amino acids $\mathrm{R}$ and $\mathrm{E}$ occur in the residues at the ' $d$ ' and ' $e$ ' positions of the MYH6 coiled-coil domain, respectively.

FIGURE 2. Structural organization of wild type (WT) FLAG-MYH6 and variant (R1822_E1823dup) in the myofibrils of differentiated $\mathrm{C} 2 \mathrm{C} 12$ cells.

MYH6 distribution was detected by anti-FLAG tag antibody (red) and nuclei were counterstained with Hoechst33342 (blue). Based on the structural organization, we grouped them into three classes. A : Striated I cells have well-defined myofibrils running parallel to each other along the long axis of the cells (ad WT n=53; ad R1822_E1823dup n=17). B : Striated II cells contain a mixture of nonstriated myofibrils interspaced with thin and misaligned striated myofibrils (ad WT $n=24$; ad R1822_E1823dup $n=28$ ). C : Nonstriated cells contain brightly fluorescent speckles and lack organized repeating units that are characteristic of myofibrils 
(ad WT n=11; ad R1822_E1823dup n=35).D : R1822_E1823dup variant significantly reduced the striated I patterns with a concomitant increase of the nonstriated pattern $\left(\chi^{2}\right.$ test; $\left.p<0.05\right)$.

FIGURE 3. Structural comparison of wildtype and mutant MYH6 proteins.

A : Comparison of the overall wildtype MYH6 (blue) and mutant MYH6 L1824delR1822_E1823dupL (pink) proteins. Wildtype helix peptide chain between 1812 and 1857 amino acids is represented by a blue molecular framework. The hydrogen bonding is represented by green dotted lines. Arg1834 in A chain $\left({ }^{\mathrm{A}}\right)$ interacts with Asn1835 in B chain $\left({ }^{\mathrm{B}}\right)$ by hydrogen bonds. Gln $1813^{\mathrm{A}}$ binds Glu1818 ${ }^{\mathrm{B}}$ by hydrogen bonds. B : The helix peptide chain containing L1824delR1822_E1823dupL between 1812 and 1857 amino acids is represented by a pink molecular framework. The insertion of residues $1823 \Delta$ and $1824 \Delta$ (wheat sticks) is connected to two flanks in the form of a random-coil, instead of an $\alpha$-helix. The insertion of the two residues further distorts the secondary structure of the two peptide chains, leading Ser $1842^{\mathrm{A}}$ and Asn $1840^{\mathrm{B}}$ to change to a random-coil. The long helix peptide chain between 1812 and 1857 amino acids is divided into three short helix regions. Arg1834 ${ }^{\mathrm{A}}-\mathrm{Asn} 1835^{\mathrm{B}}$ and $\mathrm{Gln} 1813^{\mathrm{A}}-\mathrm{Glu} 1818^{\mathrm{B}}$ lose their hydrogen bonds. Several new hydrogen bonds are generated, such as $\operatorname{Arg} 1820^{\mathrm{A}}-\mathrm{Gln} 1825^{\mathrm{B}}$, Ser1838 ${ }^{\mathrm{A}}-\mathrm{Asn} 1835^{\mathrm{B}}$ and $\operatorname{Met} 1842^{\mathrm{A}}-\operatorname{Ser} 1845^{\mathrm{B}}$. Thus, R1822_E1823dup increases the volume expansion of the dimer and impairs the $\alpha$-helix, which may increase $\alpha$-helical coiled-coil stability and limit the interaction between the coiled-coil domains.

FIGURE 4. The apoptosis and cell viability of $\mathrm{C} 2 \mathrm{C} 12$ cells was detected using flow cytometry.

A-C : After two days, the apoptosis rates of MYH6-WT $(3.16 \% \pm 1.30)$ and the MYH6- R1822_E1823dup group $(1.43 \% \pm 0.26)$ were assessed by flow cytometry. The percentage of Annexin-V positive cells in each group is shown. The results showed significant change $(p<0.05)$. D : Cell viability in the MYH6-WT group $(0.82 \% \pm 0.19)$ and MYH6- R1822_E1823dup group $(0.84 \% \pm 0.18)$ was detected by MTT. The relative optical density showed no significant difference $(p>0.05)$.

\section{Hosted file}

Table 1.pdf available at https://authorea.com/users/373694/articles/491304-novel-insertionmutation-r1822_e1823dup-in-myh6-coiled-coil-domain-causing-familial-atrial-septal-defect

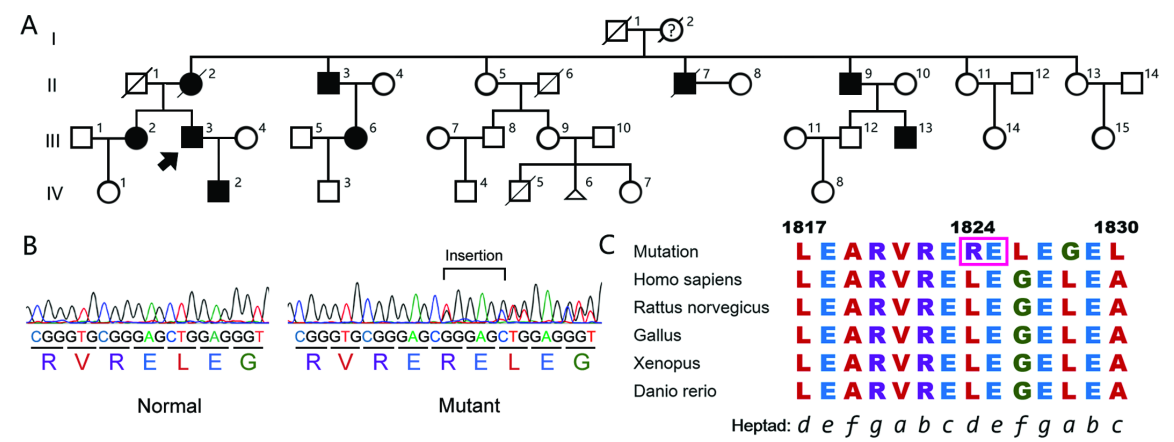



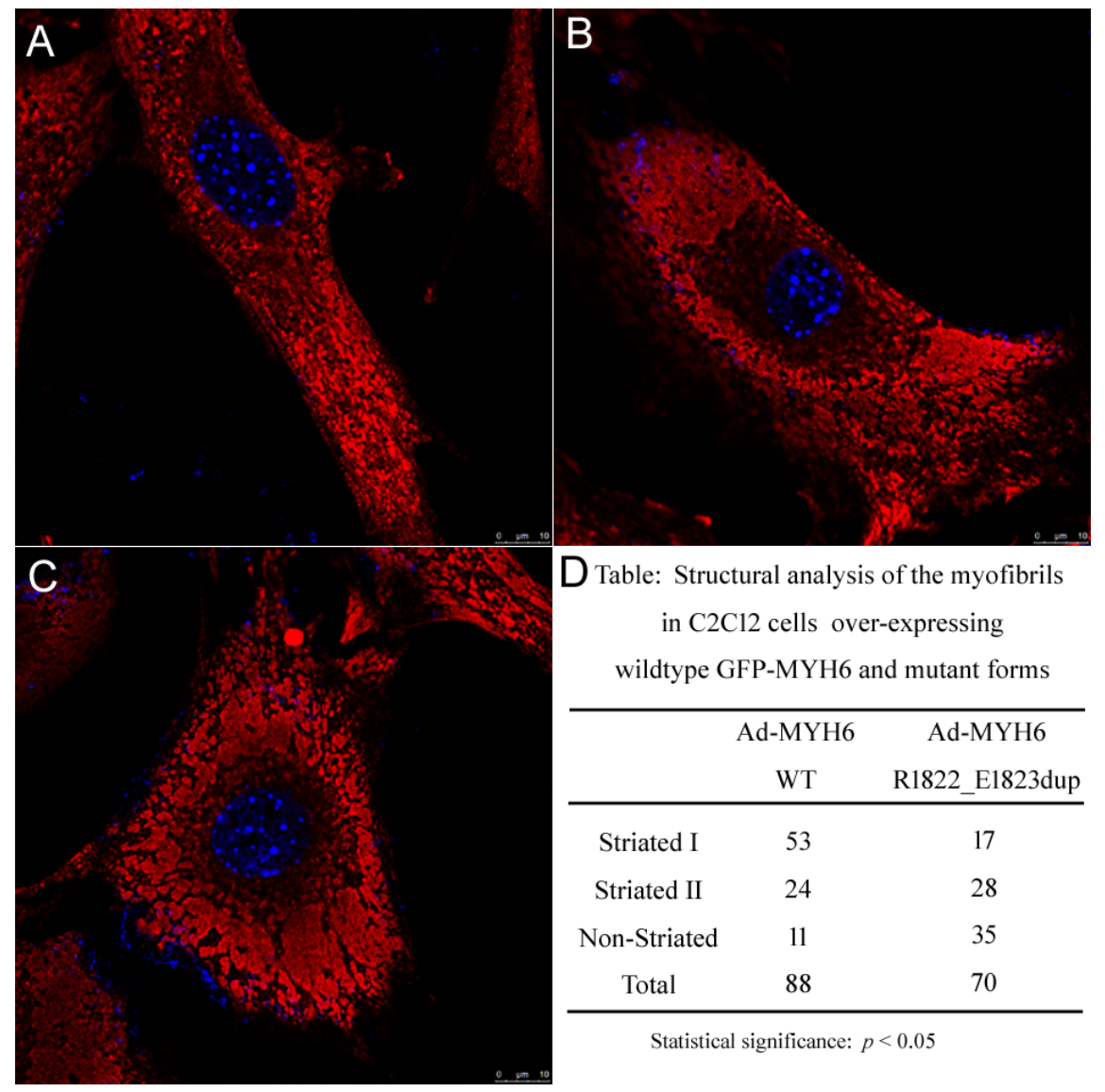

D Table: Structural analysis of the myofibrils in $\mathrm{C} 2 \mathrm{C} 12$ cells over-expressing wildtype GFP-MYH6 and mutant forms

\begin{tabular}{ccc}
\hline & $\begin{array}{c}\text { Ad-MYH6 } \\
\text { WT }\end{array}$ & $\begin{array}{c}\text { Ad-MYH6 } \\
\text { R1822_E1823dup }\end{array}$ \\
\hline Striated I & 53 & 17 \\
Striated II & 24 & 28 \\
Non-Striated & 11 & 35 \\
Total & 88 & 70 \\
\hline
\end{tabular}

Statistical significance: $p<0.05$

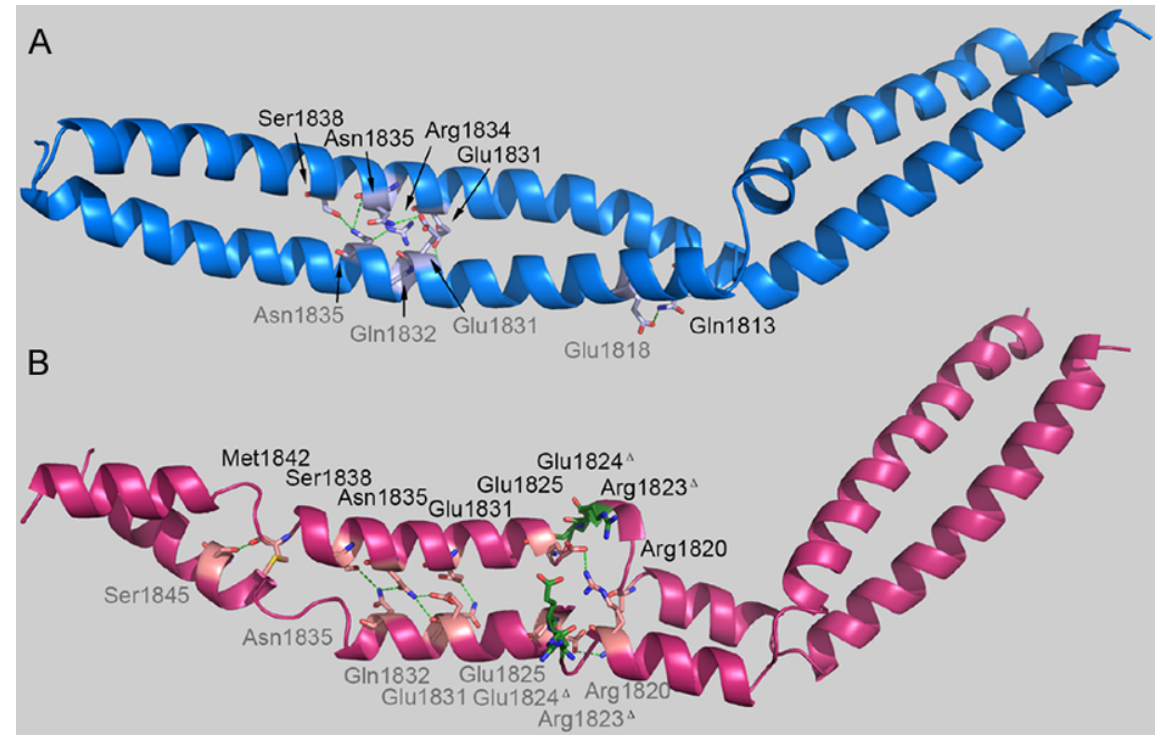



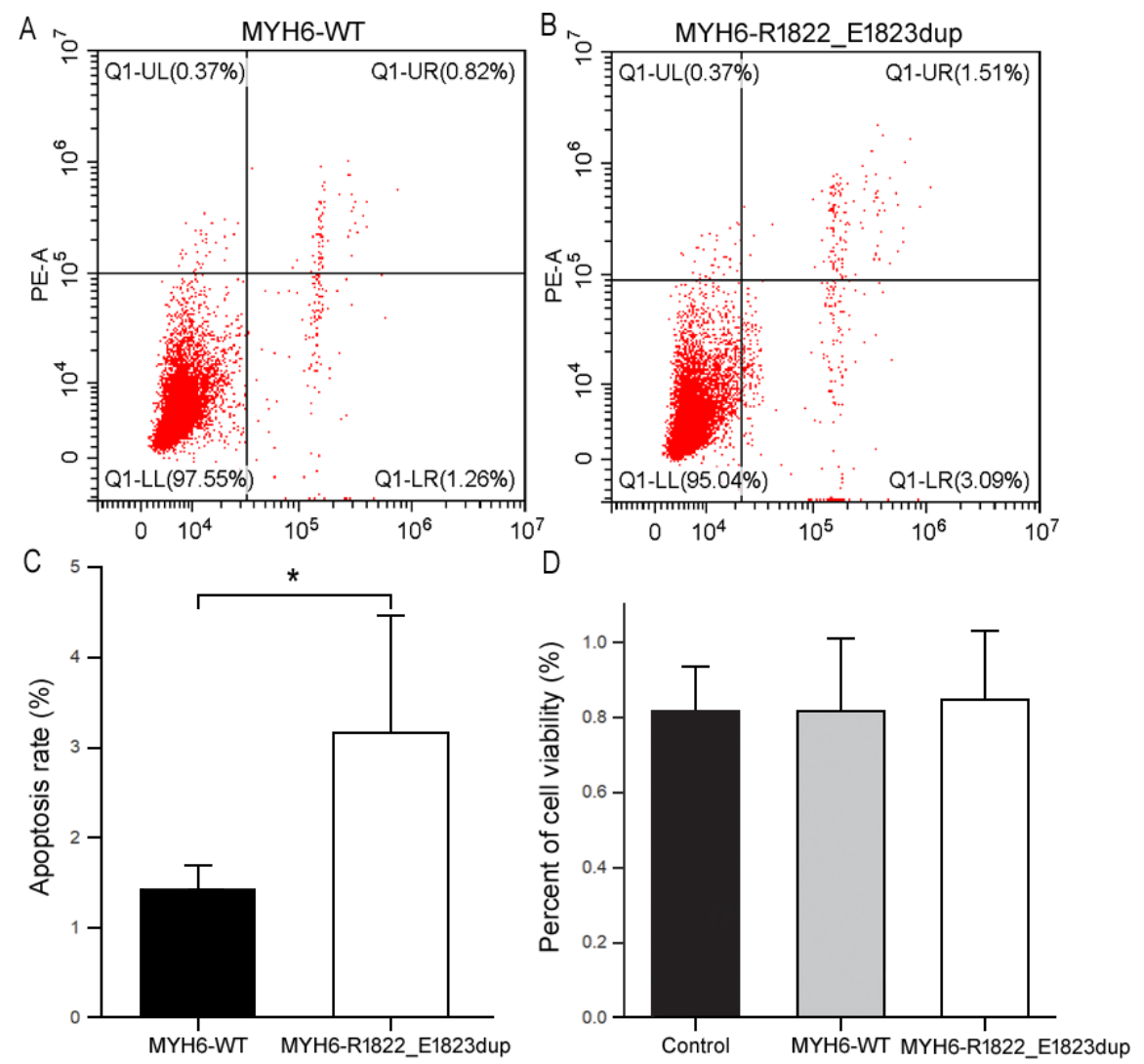